\title{
Preheating in Dirac-Born-Infeld inflation
}

\author{
Nazim Bouatta, Anne-Christine Davis and Raquel H. Ribeiro \\ e-mail: N.Bouatta, A.C.Davis and R.Ribeiro@damtp.cam.ac.uk \\ Department of Applied Mathematics and Theoretical Physics \\ Centre for Mathematical Sciences, Wilberforce Road \\ Cambridge CB3 0WA, United Kingdom
}

\section{David Seery}

\author{
e-mail: D.Seery@sussex.ac.ut \\ Astronomy Centre, University of Sussex \\ Falmer, Brighton BN1 9QH, United Kingdom
}

\begin{abstract}
We study how the universe reheats following an era of chaotic Dirac-BornInfeld inflation, and compare the rate of particle production with that in models based on canonical kinetic terms. Particle production occurs through non-perturbative resonances whose structure is modified by the nonlinearities of the Dirac-Born-Infeld action. We investigate these modifications and show that the reheating process may be efficient. We estimate the initial temperature of the subsequent hot, radiation-dominated phase.
\end{abstract}

KEYWORDS: preheating, inflation, cosmology of the very early universe. 


\section{Contents}

1. Introduction 1

2. Dirac-Born-Infeld inflation 3

2.1 Review of DBI Cosmology 3

2.2 Coupling to matter fields

3. Parametric resonance and particle production 8

3.1 Inflaton production 9

3.2 Matter particle production 11

3.3 Preheating with a symmetry breaking term

3.4 Efficiency of narrow-resonance preheating 13

4. Perturbative reheating 14

4.1 Reheating temperatures 15

5. Conclusions 16

A. Canonically normalized theory 17

B. Floquet exponents from Hill's equation 18

\section{Introduction}

Measurements of the microwave background temperature made by the WMAP satellite over the last decade [1] are consistent with the idea that structure in the universe originated as small fluctuations during a primordial era of accelerated expansion, or 'inflation'. Inflation leaves the universe in a cold vacuum state, devoid of radiation and matter. If our own universe experienced an inflationary era in its past, we must conclude that some process repopulated its sterile vacuum with the abundant quanta we see today. Much less is known about this 'reheating' process than is understood about the observationally accessible density fluctuation.

Properties of inflationary fluctuations largely decouple from the details of whatever physics drives the accelerating epoch, leaving only weak traces. ${ }^{1}$ This freedom has encouraged many proposals for the underlying microphysics, nearly all of which are equally unconstrained by observation. In an especially interesting proposal, due to Silverstein \& Tong [3] and later elaborated by Alishahiha, Silverstein \& Tong [4], inflation occurs while

\footnotetext{
${ }^{1}$ These traces are presently the subject of intense theoretical and observational effort; see, e.g. Ref. [2].
} 
a D3-brane moves in a warped background spacetime. ${ }^{2}$ As we shall discuss in $\$ 2$, the motion of the brane is controlled by an action of Dirac-Born-Infeld ("DBI") type and the resulting model is known as DBI inflation. In this paper we study the reheating era which must follow such an inflationary phase. Reheating in related brane-world models has been studied in Refs. [8, 9, 10, 11, 12]. In contrast to the primordial perturbations, reheating depends strongly on many model-specific details 13, 14. It is a subtle function of nonlinear physics, probing information complementary to the linear physics of the density fluctuation. Nonlinear properties vary widely between competing inflationary models, and there are important differences between DBI models and those with canonical kinetic terms.

Many authors have contributed to the theory of particle production, which is now well-developed [15, 16, 17, 18]. ${ }^{3}$ In perturbative reheating, the decay rate of inflaton particles into other species of matter is calculated directly from an S-matrix. These calculations assume any relics of previous decays to be sufficiently diffuse that particle production takes place into an effectively pristine vacuum. It was later understood that a Bose enhancement of the decay rate may occur for integer-spin species if the products of previous decays accumulate, leading to a resonant phase of out-of-equilibrium production known as preheating. A different effect, so-called tachyonic preheating [23, 24, 25], is associated with the end of inflation in certain models. Unlike conventional preheating this does not merely convert one species of particle into another, but (like any tachyonic instability) reflects a preference to convert one vacuum state to another by the rapid accumulation and condensation of particles.

These phenomena may occur in any effective field theory of the post-inflationary universe, whatever its microphysical origin. The general theory has subsequently been applied to concrete models, including some versions of brane inflation, in which it is possible to give a more refined interpretation [26, 27]. In these models, inflation ends when the moving D3-brane becomes close to an antibrane (a $\overline{\mathrm{D} 3}$-brane), allowing open strings stretching between the D3- $\overline{\mathrm{D} 3}$ pair to become excited. The open string states include a tachyon which induces fragmentation of the D3 brane into D0 branes, described on the D3 worldvolume by a phase of tachyonic preheating. The D0 fragments decay into closed string states, which are enumerated by the Kaluza-Klein ("KK") modes of supergravity fields in the warped background. These KK modes subsequently decay into the species of matter and radiation which populate our universe [27].

In this paper we study a version of the DBI model in which the end of inflation is less dramatic. We work in a model where the D3 comes to rest near an extremity of its warped background, referred to as the "tip of the throat." As it settles on its resting-place it executes coherent oscillations, inducing growing fluctuations which fold and wrinkle its surface. This scenario was suggested by Silverstein \& Tong [3]. Our interest is in the qualitative differences which may emerge in concrete models. Should we expect the

\footnotetext{
${ }^{2}$ D-branes are extended objects in spacetime. For a review of their properties see Johnson 疖; inflation in such models has been reviewed by McAllister \& Silverstein 66. Many detailed properties were discussed by Chen 肘.

${ }^{3}$ The theory of preheating has been reviewed by Allahverdi et al. [19], Kofman et al. [20] and Bassett et al. 21]. See also Ref. 22].
} 
reheating temperature to increase or decrease? Is parametric resonance more efficient, perhaps due to the extra non-linearities of the DBI action, or do these same non-linearities conspire to shut off the resonant channels? For this reason we focus on differences with the case of canonical kinetic terms and largely ignore similarities. For example, the use of perturbation theory in either case will break down at the onset of back-reaction, when the population of matter species grows to the point where it cannot be ignored. This affects the canonical and DBI cases equally, and we restrict our comparison to times before back-reaction becomes significant.

This paper is organized as follows. In $\$ 2$ we review the dynamics of inflation in DBI models and discuss a toy model in which inflation ends as the brane settles to its final position in the warped background. This model is comparable to chaotic inflation in a theory with canonical kinetic terms. In $\$ 3$ we discuss parametric resonance, making a comparison with canonical chaotic inflation. In $\$$ 过 we study the stage of reheating and estimate the reheating temperature. We conclude in 8 .

Throughout this paper, we work in units where $\hbar=c=1$ and set the reduced Planck mass to unity, $M_{\mathrm{P}} \equiv(8 \pi G)^{-1 / 2}=1$. The metric signature is taken to be $(-,+,+,+)$.

\section{Dirac-Born-Infeld inflation}

In $\S 2.1$ we review inflation using a Dirac-Born-Infeld Lagrangian and study the oscillating era which occurs while the brane is settling near the tip of throat. In $\$ 2.2$ we discuss possible couplings of the inflaton to matter.

\subsection{Review of DBI Cosmology}

In the scenario discussed by Silverstein \& Tong [3], one or more D3-branes move in a warped throat, taken to be an elongated region of a six-dimensional compact manifold with metric $\mathrm{d} s_{6}^{2}$. Transverse distance within the throat is measured by a parameter $\tau$, and the entire ten-dimensional geometry can be written schematically

$$
\mathrm{d} s_{10}^{2}=h^{-1 / 2}(\tau) \eta_{\mu \nu} \mathrm{d} x^{\mu} \mathrm{d} x^{\nu}+h^{1 / 2}(\tau) \mathrm{d} s_{6}^{2},
$$

where $h(\tau)$ is a warp factor. As $\tau \rightarrow \infty$ there is an ultraviolet regime where the throat is

glued onto to the rest of the $\mathrm{d} s_{6}^{2}$. In this region the throat corresponds to a cone over an Einstein manifold. In the infrared regime where $\tau \rightarrow 0$, corresponding to the "tip" of the throat, fluxes wrapped along cycles of the conifold smooth the conical singularity with an $S^{3}$ cap.

A low energy description of a D3 brane travelling in this warped throat is given by

$$
S=\frac{1}{2} \int \mathrm{d}^{4} x \sqrt{-g}\{R+2 P(\phi, X)\}
$$

where $g_{\mu \nu}$ is the pull-back of Eq. (2.1) to the brane worldvolume, and $R$ is the Ricci scalar constructed from $g_{\mu \nu}$. The worldvolume scalar $\phi$ is a collective coordinate which measures 
the radial position of the brane within the throat, and $X \equiv-g^{\mu \nu} \partial_{\mu} \phi \partial_{\nu} \phi$. The action describing evolution of $\phi$ satisfies

$$
P(\phi, X)=-f_{1}(\phi)\left[\sqrt{1-f_{2}(\phi) X}-1\right]-V(\phi),
$$

in which $f_{1}$ and $f_{2}$ are determined by the warped geometry and $V$ is a potential which describes how the brane is attracted towards the bottom of the throat. By choosing coordinates we can arrange that $f_{1}=f_{2}^{-1} \equiv f^{-1}$. In these coordinates $f^{-1}$ measures the tension of the D3-brane, redshifted by the warp factor, giving $f$ canonical dimension $\left[\mathrm{GeV}^{-4}\right]$. The proper tension $T_{3}$ is set in terms of the inverse string tension $2 \pi \alpha^{\prime}$ and the string coupling, $g_{s}$, by $T_{3}^{-1}=(2 \pi)^{3}\left(\alpha^{\prime}\right)^{2} g_{s}$. After redshifting, $f=h / T_{3}$.

If the throat is exactly anti de Sitter, the result is a static BPS state in which the Coulomb potential is quartic. Conformal invariance forbids the existence of a mass term. If the throat is cut off then conformal invariance is broken, and we expect a mass term to be generated which receives contributions from moduli stabilization and bulk fluxes 26, 28, 29, 30, 31, 32, 33. In a general compactification, a spectrum of terms will be generated including a $\phi^{3 / 2}$ term which may lead to a phase of 'ledge' or inflexion-point inflation. We assume this term is absent, or forbidden by a symmetry. Therefore, for small departures from the equilibrium point, the potential attracting the brane towards the infrared tip of the throat is quadratic [3],

$$
V(\phi)=\frac{1}{2} m^{2} \phi^{2}
$$

D-brane inflation with this potential is sometimes referred to as the 'UV model'. A related small-field ('IR') model due to Chen [7] takes $V=V_{0}-m^{2} \phi^{2} / 2$ with $V_{0}$ dominant, but in this paper we do not consider this possibility. In DBI models there is less need to tune the contributions to $m^{2}$ to avoid an $\eta$ problem [34], since inflation can be supported on steeper potentials than would be allowed with canonical kinetic terms.

As the brane drifts towards the infrared tip of the throat, $\phi$ slowly decreases to zero. In this limit $\dot{\phi}^{2} \ll 1$ and the square root can be expanded in powers of $X$, reproducing the action for a canonically normalized scalar field with potential $V$. On the other hand, if the brane is moving relativistically then $X \sim 1$ and the square root in Eq. (2.3) cannot be reduced. ${ }^{4}$ To measure the relative importance of these nonlinear terms it is conventional to define a Lorentz factor for the brane,

$$
\gamma^{-1} \equiv \sqrt{1-f(\phi) \dot{\phi}^{2}}
$$

Unitarity of Eq. (2.2) requires $\gamma$ to be real, and therefore $0 \leqslant f \dot{\phi}^{2}<1$ [38, 3], which gives a limiting velocity for $\phi$. For a slowly drifting brane we have $\gamma \sim 1$, whereas relativistic

\footnotetext{
${ }^{4}$ In this limit we retain an infinite set of non-renormalizable terms involving powers of $(\partial \phi)^{2}$ with coefficients in pre-determined ratios, but discard all operators of the form $\partial^{n} \phi$ for $n>1$. This is nevertheless sensible because $\sqrt{1-f X}$ is the unique operator containing only $\partial \phi$ which is compatible with higherdimensional isometries of the DBI model, and therefore satisfies a form of the non-renormalization theorem 3. 35, 36, 37.
} 
motion corresponds to $\gamma \gg 1$. The equations of motion for the inflaton are

$$
\ddot{\phi}+3 H \frac{\dot{\phi}}{\gamma^{2}}-\frac{f^{\prime}}{f^{2}}+\frac{3}{2} \frac{f^{\prime}}{f} \dot{\phi}^{2}+\frac{1}{\gamma^{3}}\left(\frac{f^{\prime}}{f^{2}}+V^{\prime}\right)=0
$$

where a prime ' denotes a derivative with respect to $\phi$. In the non-relativistic limit $f \rightarrow 0$ and $\gamma \rightarrow 1$, and Eq. (2.6) reduces to the Klein-Gordon equation for a canonically normalized scalar field.

Maps of the microwave background temperature anisotropy place stringent constraints on $\gamma$, which can source a large signal in the equilateral mode of the bispectrum of order $f_{\mathrm{NL}} \sim-0.32 \gamma^{2}$ 此. An analysis of WMAP 5-year data yields $f_{\mathrm{NL}}=155 \pm 140$ in this channel [39], where the quoted error is $1 \sigma$; at $95 \%$ confidence the requirement is roughly $\gamma \lesssim 18$. In this paper we will assume this upper limit will continue to be eroded, perhaps ultimately constraining $\gamma$ to be rather small, so that it is natural to imagine the model is close to its non-relativitic limit. Accordingly we suppose that inflation is approximately of the slow-roll variety. In this limit the evolution of $\phi$ is close to a canonical model, up to small corrections controlled by the deviation of $\gamma$ from unity. We will solve Eqs. (2.6)-(2.4) perturbatively in powers of these small deviations.

Inflation ends near the tip of the throat, where the $S^{3}$ cap smoothly cuts off further decrease of the warp factor 40, 41. In this region there is no redshifting associated with motion down the throat, and the brane tension $f^{-1}$ becomes approximately constant. Silverstein \& Tong [3] suggested that the relevant physics of the capped throat could be captured by approximating the tension as

$$
f(\phi) \equiv \frac{\lambda}{\left(\phi^{2}+\mu^{2}\right)^{2}}
$$

where $\lambda$ is the 't Hooft coupling and $\mu$ is the energy scale of the infrared cut-off. We have chosen the origin of $\phi$ so that the final resting place of the brane can be taken to be $\phi=0$. The warp factor corresponding to Eq. (2.7) is not itself a solution of Einstein's equations, but is qualitatively reasonable. When $\phi \ll \mu$, this becomes independent of the brane position $\phi$,

$$
f \equiv \frac{\lambda}{\mu^{4}}
$$

We will discuss natural values for $\lambda$ and $\mu$ in $\S$.

We restrict our attention to small oscillations around this position, contained entirely within the cut-off region where $f^{-1}$ is approximately constant, and suppose that $m \gg H$. Therefore many oscillations take place in one Hubble time and terms in Eq. (2.6) arising from Hubble friction can be ignored. We will justify these statements below. Making all these approximations and taking the non-relativistic limit where $\gamma=1$, coherent oscillations of the brane occur with constant amplitude $\mathcal{A}$,

$$
\phi_{0}(t) \simeq \mathcal{A} \cos m t
$$


Validity of perturbative approximation. Our analysis depends on the approximations described above, and will provide a useful description only when perturbative relativistic corrections dominate those from other sources.

When is this likely to occur? After integrating out fluxes and other UV perturbations, we are left with a low energy theory controlled by an effective potential for the D-brane [32, 33], of the form $V=\sum_{i} c_{i} m^{4-\Delta_{i}} \phi^{\Delta_{i}}$, where the $c_{i}$ are expected to be $\mathrm{O}(1)$ in a generic compactification. The mass $m$ is an ultraviolet scale which determines where the throat is glued onto the main bulk of the compactification manifold. The leading term corresponds to $\Delta=3 / 2$. Assuming some symmetry forces this to be absent, the next $\Delta=2$ term will typically dominate higher contributions when $\phi \ll m$.

The infrared cutoff scale, $\mu$, must satisfy $\mu<m$. Therefore, provided we take $\phi<\mu$, in order that we are safely within a region where the warp factor is constant, we can expect corrections from both features in the throat geometry and higher-order terms in the D-brane potential to be negligible.

Now consider our neglect of Hubble drag in Eq. (2.9). In practice, Hubble drag would cause $\mathcal{A}$ to decrease slowly, roughly like $(m t)^{-1}$ [42]. Suppose $H_{f}$ and $H_{i}$ are two values of the Hubble parameter separated by $N$ oscillations. Since the brane worldvolume experiences a matter-dominated era while the coherent oscillations are ongoing, these values are related by

$$
\frac{H_{i}}{H_{f}} \sim 1+A N \frac{H_{i}}{m}
$$

where $A$ is a constant of order unity. The corresponding oscillation amplitudes are

$$
\frac{\mathcal{A}_{i}}{\mathcal{A}_{f}} \sim 1+A^{\prime} N \frac{\mathcal{A}_{i}}{M_{\mathrm{P}}}
$$

in which $A^{\prime}$ is a different constant, also of order unity. Unless $N \gg 1$, our assumption that $m \gg H$ is sufficient to ensure $H_{i} \approx H_{f}$. In addition, Eq. (2.11) shows that $\mathcal{A}_{i} \ll M_{\mathrm{P}}$ is sufficient to ensure $\mathcal{A}_{i} \approx \mathcal{A}_{f}$. In view of the requirement $\mathcal{A} \ll m$ required to ensure the quadratic potential is an adequate approximation, where $m$ is typically of order the infrared cutoff scale, this is amply satisfied. Therefore, in what follows, we will restrict our attention to $N<10^{2}$ oscillations and neglect the time dependence of $H$ and $\mathcal{A}$.

Relativistic corrections to background solution. Corrections to the non-relativistic motion described by (2.9) will be perturbative provided $\left|f \dot{\phi}_{0}^{2}\right| \ll 1$. Introducing a dimensionless positive quantity $\epsilon$, satisfying

$$
\epsilon \equiv f m^{2} \mathcal{A}^{2}
$$

this perturbative condition can be rewritten $\epsilon \ll 1$. We must nevertheless work in a regime where $\epsilon \gtrsim \phi / m$ or $\mu / m$, in order that the small relativistic corrections we obtain are not dominated by others which we do not calculate. We should trust our analysis for perhaps $\epsilon \sim 10^{-1}$ to $10^{-3}$, but not for significantly larger or smaller $\epsilon$.

To go further, we must determine the leading $\mathrm{O}(\epsilon)$ corrections to (2.9). Note that $\epsilon$ is not the conventional slow-roll parameter, given by $-\dot{H} / H^{2}$, and these corrections are 
not related to the slow-roll approximation. The governing equation can be obtained by dropping Hubble friction terms in Eq. (2.6), yielding

$$
\ddot{\phi}+\left(1-\frac{3}{2} f \dot{\phi}^{2}\right) \frac{\partial V}{\partial \phi} \simeq 0
$$

It is tempting to imagine that Eq. (2.13) can be solved perturbatively, writing $\phi=\phi_{0}+\delta \phi$ with $\delta \phi$ of order $\mathrm{O}(\epsilon)$. However, Eq. (2.13) is rather nonlinear and also induces corrections to the fundamental frequency $m$. To capture these effects, consider a WKB-type solution of the form $\phi=\mathcal{A} \cos \theta(t)$ and solve for the phase $\theta(t)$ to order $\mathrm{O}(\epsilon)$. To match smoothly with (2.9) in the limit $\epsilon \rightarrow 0$, we must have $\theta(t)=m t+\delta \theta(t)$. From Eq. (2.13) we conclude that $\delta \theta(t)$ must satisfy

$$
\delta \ddot{\theta}+2 m(\cot m t) \delta \dot{\theta}+\frac{3 m^{2} \epsilon}{2} \sin m t \cos m t=0 .
$$

Dropping an unwanted divergent contribution, ${ }^{5}$ the solution is

$$
\phi(t)=\mathcal{A} \cos \left\{m t\left(1-\frac{3 \epsilon}{16}\right)+\frac{3 \epsilon}{32} \sin 2 m t\right\} .
$$

Corrections to the amplitude are also calculable in principle, but need not be computed if we are ignoring the time dependence of $\mathcal{A}$.

In comparison with the canonical solution, the phase shows a secular drift proportional to $\epsilon m t$. Although the calculation remains under perturbative control, this drift generates order unity deviations from the non-relativistic background after $1 / \epsilon$ oscillations. There is also a superposed phase oscillation, proportional to $|\epsilon \sin 2 m t| \ll 1$, which always generates small corrections. To make contact with the canonical solution, 2.15) can be written in the alternate form

$$
\phi(t)=\mathcal{A}\left\{1-\frac{3 \epsilon}{16} \sin ^{2}\left[m t\left(1-\frac{3 \epsilon}{16}\right)\right]\right\} \cos m t\left(1-\frac{3 \epsilon}{16}\right),
$$

which is equivalent to (2.15) up to $\mathrm{O}(\epsilon)$. Eq. (2.16) exhibits the first perturbative relativistic corrections to the motion of the brane: first, a frequency shift in the background motion $\phi_{0}$, corresponding to $m \mapsto m(1-3 \epsilon / 16)$; and second, a small periodic fluctuation superposed over the background oscillations.

\subsection{Coupling to matter fields}

The reheating phase is designed to fill the universe with Standard Model degrees of freedom. Pauli's exclusion principle usually prevents efficient preheating into fermions [43], so we suppose the inflaton $\phi$ couples to bosonic modes which are taken to have spin zero. The resulting scenario is frequently adopted in the literature, and gives an acceptable phenomenology subsequently decay to fermions is efficient. We treat the couplings between

\footnotetext{
${ }^{5}$ This mode is proportional to cot $m t$, which is divergent at $t=0$. It is projected out by the boundary conditions, and therefore does not lead to an instability.
} 
the inflaton and the matter modes phenomenologically, denoting the matter field $\chi$ and taking it to be described by the low energy effective Lagrangian

$$
\mathcal{L} \supseteq-\frac{1}{2} \partial_{\mu} \chi \partial^{\mu} \chi-\frac{1}{2} m_{\chi}^{2} \chi^{2}-\frac{1}{2} g^{2} \phi^{2} \chi^{2}
$$

where $g$ is a dimensionless coupling and $m_{\chi}$ is a bare mass. The symbol ' $\supseteq$ ' is used to indicate that the Lagrangian contains these contributions among others. We do not claim that such interactions necessarily arise in string models, although we hope the resulting phenomenology will be representative of those that do. The equation of motion for $\chi$ is

$$
\ddot{\chi}+3 H \dot{\chi}-\frac{1}{a^{2}} \nabla^{2} \chi+\left(m_{\chi}^{2}+g^{2} \phi^{2}\right) \chi=0,
$$

where $\nabla^{2}$ is the spatial coordinate Laplacian. Matter production in this model is studied in $\$ 3.2$.

In $\S 3.3$ we will consider an alternative model, in which the matter field $\chi$ is coupled to a symmetry-breaking potential of the form

$$
V(\phi, \chi)=\frac{1}{2} m^{2}(\phi-\sigma)^{2}+\frac{1}{2} g^{2} \phi^{2} \chi^{2}
$$

This causes the brane to be attracted to a radial position for which $\phi=\sigma$, representing a toy model of compactifications in which the mobile D-branes are brought to rest at a finite point in the throat.

\section{Parametric resonance and particle production}

As the brane settles towards its final resting place, its coherent oscillations lead to copious non-perturbative particle production. We briefly review this process, with the aim of fixing our notation.

To quantize any field, such as the inflaton $\phi$ or the matter field $\chi$, we pick two independent solutions to the mode equation,

$$
\ddot{\chi}_{k}+3 H \dot{\chi}_{k}+\left(k^{2}+m_{\chi}^{2}+g^{2} \phi^{2}\right) \chi_{k}=0,
$$

where $k$ is the comoving wavenumber, and to be concrete we have chosen to frame our discussion for $\chi$ although an equivalent construction can be given for $\phi$. Label these solutions $\chi_{k}^{\mathrm{cl}}$ and $\chi_{k}^{\mathrm{cl} *}$. The Heisenberg field corresponding to $\chi$ can be written

$$
\chi(t, \mathbf{x})=\int \frac{\mathrm{d}^{3} k}{(2 \pi)^{3}}\left(\chi_{k}^{\mathrm{cl}} a_{k}^{\dagger}+\chi_{k}^{\mathrm{cl} *} a_{-k}\right) \mathrm{e}^{\mathrm{i} \mathbf{k} \cdot \mathbf{x}}
$$

in which we have assumed that the normalisation of $\left\{\chi_{k}^{\mathrm{cl}}, \chi_{k}^{\mathrm{cl} *}\right\}$ has been adjusted so that $a_{k}$ and $a_{k}^{\dagger}$ obey the usual creation-annihilation algebra,

$$
\left[a_{k}, a_{k^{\prime}}^{\dagger}\right]=(2 \pi)^{3} \delta\left(\mathbf{k}-\mathbf{k}^{\prime}\right)
$$

What is the occupation number $n_{k}$ for momenta of magnitude $k$ ? The energy density, $E_{k}$, associated with $\chi$-quanta of wavenumber $k$ satisfies $2 E_{k}=\left|\dot{\chi}_{k}^{\mathrm{cl}}\right|^{2}+\omega_{k}^{2}\left|\chi_{k}^{\mathrm{cl}}\right|^{2}$, where 
$\omega_{k}^{2}=k^{2}+g^{2} \phi^{2}$. Accounting for subtraction of the energy density associated with vacuum fluctuations, when the occupation number is large it must be given to a good approximation by

$$
n_{k} \approx \frac{E_{k}}{\omega_{k}}=\frac{\omega_{k}}{2}\left(\frac{\left|\dot{\chi}_{k}^{\mathrm{cl}}\right|^{2}}{\omega_{k}^{2}}+\left|\chi_{k}^{\mathrm{cl}}\right|^{2}\right)-\frac{1}{2} .
$$

It follows that the occupation number can be determined from knowledge of the mode functions $\chi_{k}^{\mathrm{cl}}$ and their derivatives; when $\left|\chi_{k}^{\mathrm{cl}}\right|$ or $\left|\dot{\chi}_{k}^{\mathrm{cl}}\right|$ are large, we expect large occupation numbers. In what follows we shall not need to make use of the quantum field $\chi$, which allows us to drop the label 'cl' on each mode function. In particular, if $\chi_{k}$ experiences exponential growth - implying that the production of $\chi$ particles is stimulated by the presence of existing $\chi$-quanta - then Eq. (3.4) shows we can expect $n_{k}$ to attain macroscopic values very rapidly. This is the phenomenon of preheating, discussed in $\oint_{1}$.

To determine when $\chi_{k}$ grows, it is often useful to translate the mode equation for $\chi_{k}$ into a version of Mathieu's equation or its generalizations. The classical Mathieu equation is 44, 45

$$
\frac{\mathrm{d}^{2} \chi_{k}}{\mathrm{~d} z^{2}}+\left(A_{k}-2 q \cos 2 z\right) \chi_{k}=0
$$

where we have introduced a dimensionless time $z$ to be defined below. In the following sections we give explicit expressions for $A_{k}$ and $q$ applicable to both inflaton and matter fluctuations. For certain combinations of these parameters the solutions of Mathieu's equation exhibit exponential growth. If $q \gtrsim A_{k}$, the effective mass can become temporarily zero. In this case it is energetically inexpensive to copiously produce $\chi$-quanta, referred to as broad resonance. Alternatively, if $q \ll A_{k}$ then the effective mass never passes through zero, and the combinations which yield exponential growth occur only in narrow bands of momentum space. This scenario is known as narrow resonance. These bands are centred on momenta for which $A_{k}=\ell^{2}$, where $\ell \in \mathbb{N}$, and roughly correspond to values of $k$ for which $\left|A_{k}-\ell^{2}\right| \lesssim q^{\ell}$. If $|q| \ll 1$ then at large $\ell$ these bands become increasingly narrow.

\subsection{Inflaton production}

The foregoing analysis applies equally to inflaton and matter fluctuations. First, consider the inflaton mode of wavenumber $k$. We set $\phi$ equal to its background value, denoted $\varphi$, which is determined up to first-order relativistic corrections by (2.16). At the onset of oscillations it is reasonable to assume that $\chi$ is in its vacuum state $\chi=0$. Until the accumulation of $\chi$-quanta causes the background $\chi$ field to grow, the inflaton fluctuation $\delta \phi_{k}$ evolves according to the Klein-Gordon equation, to linear order in $\epsilon$,

$$
\delta \ddot{\phi}_{k}-3 \frac{f m^{2} \varphi \dot{\varphi}}{\gamma} \delta \dot{\phi}_{k}+\left(\frac{m^{2}}{\gamma^{3}}+k^{2}\right) \delta \phi_{k}=0 .
$$

We expand uniformly to leading order in $f \dot{\varphi}^{2}$ and make the change of variables $\delta \phi_{k}=\xi y_{k}$, choosing $\xi$ to eliminate the friction term in (3.6),

$$
\frac{\mathrm{d} \ln \xi}{\mathrm{d} t}=\frac{3}{2} f m^{2} \varphi \dot{\varphi}
$$


The solution to this differential equation is

$$
\xi=\xi_{0} \exp \left\{\frac{3}{2} f m^{2}\left[\varphi^{2}(t)-\varphi^{2}\left(t_{0}\right)\right]\right\}
$$

where $\xi=\xi_{0}$ at $t=t_{0}$. This is an effective friction, or 'braking,' which could be interpreted as a perturbative manifestation of the D-cceleration mechanism [3]. It is a novel effect in the DBI scenario, with no analogue for canonical kinetic terms. Accordingly, the braking disappears in the limit $\epsilon \rightarrow 0$. The remaining time dependence is carried by $y_{k}$, which satisfies a Mathieu equation with $A_{k}$ and $q$ determined, to leading order in $\epsilon$, by

$$
A_{k}=\left(1+\frac{k^{2}}{m^{2}}\right)\left(1+\frac{3 \epsilon}{8}\right)-\frac{3 \epsilon}{4} \quad \text { and } \quad q=\frac{3 \epsilon}{8} .
$$

The associated dimensionless time $z$, which was used to write Eq. (3.5), satisfies $z=$ $m t(1-3 \epsilon / 16)$. Evidently $q \ll A_{k}$, giving resonance only in narrow bands. The $\ell=1$ band is centred at momentum $k_{1}$, given by

$$
k_{1}=\frac{m}{2} \sqrt{\frac{3 \epsilon}{2}} .
$$

According to Floquet's theory each mode behaves like $\mathrm{e}^{\mu_{k} z}$. In the $\ell=1$ band, the Floquet exponent $\mu_{k}$ has positive real solutions which correspond to exponential growth [44]

$$
\mu_{k}^{2} \simeq\left(\frac{q}{2}\right)^{2}-\left(A_{k}^{1 / 2}-1\right)^{2}
$$

This exponent is maximal for the central value $k=k_{1}$ and decreases for larger or smaller $k$, forming a band of width $\Delta_{1}$,

$$
\Delta_{1}=\frac{m \sqrt{3 \epsilon}}{2} \frac{\sqrt{2}-1}{\sqrt{2}}
$$

At the centre of the band, the Floquet exponent takes the value $\mu_{1}$

$$
\mu_{1}=\max _{\ell=1 \text { band }} \mu_{k}=\frac{3 \epsilon}{16} .
$$

Up to this point, we have written our formulae in terms of the mass, $m$, and coupling, $g$, which appear in the Lagrangian. However, these quantities only parametrize the theory. To make a meaningful comparison between the DBI model and the case of canonical kinetic terms, we must express our answer in terms of a measurable mass and coupling. We denote these measurable quantities $m_{\star}$ and $g_{\star}$, to distinguish them from the Lagrangian parameters. In the DBI theory the Lagrangian parameters have no direct physical significance, whereas in the case of canonical kinetic terms they coincide with the measurable mass and coupling at tree-level.

We determine the relationships $m_{\star}(m)$ and $g_{\star}(g)$ in Appendix A. Collecting the results of Eqs. (3.8) and (3.11), and expressing the answer in terms of $m_{\star}$ and $g_{\star}$, we find that up 
to an overall normalization near the centre of the resonant band, the inflaton modes grow like

$$
\delta \phi_{k} \sim \exp \left\{\frac{3 \epsilon}{8} \cos \left[2 m_{\star} t\left(1-\frac{7 \epsilon}{16}\right)\right]+\frac{3 \epsilon}{16} m_{\star} t\right\} .
$$

The undetermined normalization absorbs the constant $\xi_{0}$ in (3.8).

At early times, the DBI friction and the resonant growth compete, and the argument of the exponential in Eq. (3.14) can even become negative. After a few oscillations, however, the cosine which represents the friction effect becomes negligible in comparison with the resonant term.

\subsection{Matter particle production}

Our ultimate interest lies with the production of matter particles. Consider the potential (2.17). For any particle species which gains a mass at energies much less than the inflationary scale it will typically be a good approximation to assume $m_{\chi}$ is negligible in comparison with $g \phi$ unless the amplitude of oscillations is extremely small. Therefore each $\chi$-mode receives an effective mass of order $k^{2}+g^{2} \phi^{2}$. As in $\S 3.1$ we assume that preheating begins in the vacuum $\chi=0$, which will be valid until the accumulating $\chi$-quanta backreact on the zero mode. In this vacuum, small fluctuations grow according to a Hill equation of type

$$
\delta \chi_{k}^{\prime \prime}+\left(\theta_{0}+2 \theta_{2} \cos 2 z+2 \theta_{4} \cos 4 z\right) \delta \chi_{k}=0,
$$

where the parameters $\theta_{0}, \theta_{2}$ and $\theta_{4}$ obey

$$
\begin{aligned}
& \theta_{0}=\frac{k^{2}}{m^{2}}\left(1+\frac{3 \epsilon}{8}\right)+\frac{g^{2} \mathcal{A}^{2}}{2 m^{2}}\left(1+\frac{9 \epsilon}{32}\right) \\
& \theta_{2}=\frac{g^{2} \mathcal{A}^{2}}{4 m^{2}}\left(1+\frac{3 \epsilon}{8}\right) \\
& \theta_{4}=\frac{3}{128} \frac{\mathcal{A}^{2}}{m^{2}} \epsilon g^{2},
\end{aligned}
$$

and (as in $\$ 3.1$ ) a prime ' denotes a derivative with respect to $z=m t(1-3 \epsilon / 16)$. The solutions of Hill's equation grow and decay with characteristic Floquet exponent $\mu_{k}$ in a way entirely analogous to the solutions of Mathieu's equation. The exponentially growing solutions organize themselves into bands, characterized by integers $\ell$. Using the arguments of Floquet theory one can obtain an estimate of $\mu_{k}$ for the $\ell=1$ band (see Appendix $B$ ),

$$
\mu_{k}^{2} \approx\left(\frac{\theta_{2}}{2}\right)^{2}-\left(\theta_{0}^{1 / 2}-1\right)^{2} \mp \frac{\theta_{2}}{3}\left(\frac{\theta_{4}}{2}\right)^{2} \mp \frac{\theta_{2}^{3}}{2} .
$$

We believe this expression has not previously appeared in the preheating literature. In the region where our approximations are valid, we have $\mathcal{A} \ll m$. Therefore, the terms involving $\theta_{4}^{2}$ and $\theta_{2}^{3}$ are negligible. Under these conditions the Floquet exponent can be simplified, yielding

$$
\mu_{k}^{2} \approx\left(\frac{\theta_{2}}{2}\right)^{2}-\left(\theta_{0}^{1 / 2}-1\right)^{2}
$$


The centre of the $\ell=1$ band lies at $k=k_{1}$, for which $\theta_{0}\left(k_{1}\right)=1$,

$$
k_{1}^{2}=m^{2}-\frac{g^{2} \mathcal{A}^{2}}{2}-\frac{3 \epsilon}{8}\left(m^{2}-\frac{g^{2} \mathcal{A}^{2}}{8}\right) .
$$

Collecting terms as before, it follows that the unstable matter modes grow at a rate determined by

$$
\delta \chi_{k} \sim \exp \left[\left.\mu_{k}\right|_{\text {max }} m_{\star} t\left(1-\frac{3 \epsilon}{16}\right)\right] \sim \exp \left[\frac{1}{8}\left(\frac{g_{\star} \mathcal{A}}{m_{\star}}\right)^{2} m_{\star} t\left(1+\frac{3 \epsilon}{16}\right)\right] .
$$

This displays a slight enhancement compared to the equivalent canonical model.

\subsection{Preheating with a symmetry breaking term}

Now consider the symmetry-breaking potential given in Eq. 2.19). It is convenient to redefine $\phi$ so that it describes excitations around the true vacuum at $\phi=\sigma$. After making the shift $\phi \rightarrow \phi+\sigma$, the potential can be written

$$
V(\phi, \chi)=\frac{1}{2} m^{2} \phi^{2}+\frac{1}{2} g^{2} \phi^{2} \chi^{2}+g^{2} \sigma \phi \chi^{2}+\frac{1}{2} g^{2} \sigma^{2} \chi^{2} .
$$

Excitations of $\chi$ around this minimum acquire an effective mass $m_{\text {eff }}^{2}(\phi)=m^{2}+g^{2} \chi^{2}$.

Making the same assumption that preheating begins while $\chi \approx 0$, the inflaton modes will behave as in Eq. (3.14) until the growth of $\chi$ occupation numbers causes the $\chi$ zero mode to grow. Until that time, the $\chi$ modes obey

$$
\delta \ddot{\chi}_{k}+\left(k^{2}+g^{2} \phi^{2}+2 g^{2} \sigma \phi+g^{2} \sigma^{2}\right) \delta \chi_{k}=0 .
$$

We will suppose the brane executes oscillations around $\phi=\sigma$ with amplitude $\mathcal{A} \ll \sigma$, so that it can reasonably be said to have settled at its final position. Assuming that $\sigma \ll m / g$, and again neglecting any bare mass for $\chi$, fluctuations in the matter field will be controlled by a Hill equation,

$$
\delta \chi_{k}^{\prime \prime}+\left[\theta_{0}+2 \theta_{2} \cos 2 z+2 \theta_{6} \cos 6 z\right] \delta \chi_{k} \simeq 0,
$$

in which $2 z=m t(1-3 \epsilon / 16)$ and the parameters $\theta_{0}, \theta_{2}$ and $\theta_{4}$ obey

$$
\begin{aligned}
& \theta_{0}=\frac{4}{m^{2}}\left(k^{2}+g^{2} \sigma^{2}\right)\left(1+\frac{3 \epsilon}{8}\right), \\
& \theta_{2} \simeq 4 \frac{g \sigma}{m} \frac{g \mathcal{A}}{m}\left(1+\frac{21 \epsilon}{64}\right), \\
& \theta_{6} \simeq \frac{3 \epsilon}{16} \frac{g \sigma}{m} \frac{g \mathcal{A}}{m} .
\end{aligned}
$$

To determine the growth of each $\chi$-mode we require an estimate of the Floquet exponent associated with Eq. (3.23), which is again derived in Appendix B. For the $\ell=1$ band, it is given by

$$
\mu_{k} \simeq \sqrt{\left(\frac{\theta_{2}}{2}\right)^{2} \mp \frac{\theta_{2} \theta_{6}^{2}}{32} \mp\left(\frac{\theta_{2}}{2}\right)^{3}-\left(\theta_{0}^{1 / 2}-1\right)^{2}} .
$$


As above, the terms involving $\theta_{6}^{2}$ and $\theta_{2}^{3}$ can be neglected. The result is equivalent to a Mathieu equation with coefficients $A_{k}$ and $q$ satisfying

$$
A_{k}=\frac{4}{m^{2}}\left(k^{2}+g^{2} \sigma^{2}\right)\left(1+\frac{3 \epsilon}{8}\right) \quad \text { and } \quad q=\theta_{2} \simeq 4 \frac{g \sigma}{m} \frac{g \mathcal{A}}{m}\left(1+\frac{21 \epsilon}{64}\right) \ll A_{k} .
$$

Since $q$ is much smaller than $A_{k}$, we expect resonance to be of the narrow type if it occurs at all. The first instability band occurs when $A_{k}=1$ and is centred at wavenumber $k=k_{1}$, where

$$
k_{1} \simeq \frac{m}{2}\left(1-\frac{3 \epsilon}{16}\right),
$$

which is shifted by $\mathrm{O}(\epsilon)$ in comparison with the canonical case. Eqs. (3.19) and (3.27) show that the $\ell=1$ resonance injects energy into a narrow band of modes with momenta $k \sim m$. Kofman, Linde \& Starobinksy interpreted the narrowness of this resonance as multiple, rapid copies of a decay process in which a single inflaton particle of mass $\sim m$ decays into two $\chi$-particles with opposite momenta of magnitude $\sim k_{1}$ [13]. The width of the resonant band satisfies

$$
\Delta k_{1} \simeq 2 m \frac{g \sigma}{m} \frac{g \mathcal{A}}{m}\left(1+\frac{9 \epsilon}{64}\right) .
$$

Using the appropriate limit of Eq. 3.25) to estimate $\mu_{k}$, or applying (3.11), we conclude that there is a small enhancement of preheating efficiency owing to the relativistic DBI correction. Near the centre of the $\ell=1$ band, the $\chi$-modes grow like

$$
\delta \chi_{k} \sim \exp \left(\left.\mu_{k}\right|_{\max } z\right) \sim \exp \left[\frac{g_{\star} \sigma}{m_{\star}} \frac{g_{\star} \mathcal{A}}{m_{\star}} m_{\star} t\left(1+\frac{9 \epsilon}{64}\right)\right],
$$

in which the growth rate is augmented with respect to the non-relativistic or canonical cases by an $\mathrm{O}(\epsilon)$ term.

\subsection{Efficiency of narrow-resonance preheating}

The resonances we have discussed above are narrow, and therefore occupation numbers grow only for a limited set of momenta. This process is much less efficient than broad resonance, where a large range of wavenumbers experience growing occupation numbers. Nevertheless, because growth is exponential within the unstable bands, preheating by narrow resonance may still lead to an acceptably rapid conversion of inflaton modes into matter species. Gravitational redshift will cause modes of fixed comoving wavenumber to drift slowly through each unstable band from ultraviolet to infrared, and significant particle production will occur only when modes remain within an unstable band for sufficiently long that multiple e-foldings of stimulated emission occur [18]. If wavenumbers move through the unstable bands too rapidly then little stimulated emission occurs, and the resonance is washed out.

The arguments of Floquet's theory show that in each resonant band, the inflaton and matter fluctuations of wavenumber $k$ grow like $\mathrm{e}^{\mu_{k} z}$. Let us focus on the broadest instability band, which corresponds to $\ell=1$ and will generally dominate the particle production. This band has width $\sim q$, so each wavenumber remains within the unstable band for a time of 
order $q / H$. The resonance will be stable against Hubble expansion if $\left(\mu_{k} z / t\right) q / H \gtrsim 1$. It follows that preheating will drain energy from the inflaton provided

$$
\sqrt{\frac{2 H}{m_{\star}}} \lesssim \frac{3 \epsilon}{8}
$$

For the matter field, preheating will operate in the cases $\sigma=0$ and $\sigma \neq 0$ whenever

$$
\sqrt{\frac{2 H}{m_{\star}}} \lesssim \frac{1}{4}\left(\frac{g_{\star} \mathcal{A}}{m_{\star}}\right)^{2}\left(1+\frac{13 \epsilon}{32}\right)
$$

or

$$
\sqrt{\frac{H}{m_{\star}}} \lesssim 2\left(\frac{g_{\star} \sigma}{m_{\star}}\right)\left(\frac{g_{\star} \mathcal{A}}{m_{\star}}\right)\left(1+\frac{23 \epsilon}{64}\right)
$$

apply, respectively.

\section{Perturbative reheating}

While preheating is occurring it remains possible for inflaton particles to decay into other species via perturbative processes [46]. Indeed, these processes are necessary to thermalize the post-inflationary universe and bring reheating to an end.

In the theories we have been considering, such decays are allowed by the symmetrybreaking potential studied in $\S 3.3$, where there is an effective interaction $g^{2} \sigma \phi \chi^{2}$ in the true vacuum which permits the decay $\phi \rightarrow \chi \chi$. The simple potential (2.17) allows only two-body scattering and annihilation processes in vacuum. However, in the presence of an oscillating background field we can take the decay rate to be similar to that produced by the $g^{2} \sigma \phi \chi$ interaction with $\sigma \sim \mathcal{A}$. If the inflaton has direct couplings to fermionic species then perturbative production can proceed via Yukawa interactions such as $h \phi \bar{\psi} \psi$, where $\psi$ is a Dirac fermion and $\bar{\psi}=\mathrm{i} \psi^{\dagger} \gamma^{0}$ its adjoint. In this case we must suppose that any interactions in which the inflaton participates are not sufficient to spoil the possibility of successful inflation.

In this section we will continue to assume that the inflaton mass is much larger than the bare mass of $\chi$, or the mass of any fermionic species where such couplings exist. Under these conditions, the tree-level decay rates for $\phi \rightarrow \chi \chi$ and $\phi \rightarrow \bar{\psi} \psi$ can be calculated straightforwardly. The necessary expressions were given by Kofman et al. ${ }^{6}$ [18]

$$
\Gamma(\phi \rightarrow \chi \chi)=\frac{g_{\star}^{4} \sigma^{2}}{8 \pi m_{\star}}, \quad \text { and } \quad \Gamma(\phi \rightarrow \bar{\psi} \psi)=\frac{h_{\star}^{2} m}{8 \pi},
$$

where $h_{\star}$ is the measured Yukawa coupling, determined by matching Eq. (4.1) to observation. Can perturbative decays give a contribution which is competitive with preheating? In one oscillation, perturbative decays populate the $\chi$ field at a rate $\Gamma / m_{\star}$. In the same time interval preheating increases the population at a rate $\mu_{k}$. Therefore perturbative decays dominate if

$$
\Gamma \gtrsim m_{\star} \mu_{k} .
$$

\footnotetext{
${ }^{6}$ Modifications to these rates and cross-sections in a DBI model are studied in Appendix A.
} 
Let us suppose that perturbative decays dominate the production of matter species. These processes are relatively slow and it is reasonable to assume that the decay products thermalize. After thermalization has gone to completion we can expect the resulting radiation dominated universe to have a temperature $T_{R}$, defined by

$$
T_{R} \simeq 0.2 \sqrt{\Gamma M_{P}}
$$

where $\Gamma$ is the rate of the dominant decay channel.

Let us briefly recapitulate the role of perturbative processes in canonical inflation. Inflaton decays $\phi \rightarrow \chi \chi$ can become the dominant channel for inflaton decay only in the case of symmetry breaking, for which $\sigma \neq 0$ [18]. These decays begin to dominate when the amplitude of inflaton oscillations has dropped to $\mathcal{A} \sim g_{\star}^{2} \sigma / 8 \pi$. If we suppose that the curvature perturbation synthesized during the inflationary phase, denoted $\zeta$, was dominated by fluctuations in the inflaton, then we must conclude $m \sim 10^{-5} M_{\mathrm{P}}$ in order to match observation. This assumption is minimal but not mandatory; if $\zeta$ receives a dominant contribution from other sources, a wider range of masses $m$ can be tolerated. Supposing further that the coupling $g_{\star}$ has a natural value $g_{\star} \sim 10^{-1}$, we find a reheating temperature of order the symmetry breaking scale

$$
T_{R} \simeq \sigma
$$

Alternatively, it may happen that the dominant decay is into fermions. If so, Eq. (4.2) implies that the Yukawa coupling must satisfy

$$
h_{\star}^{2} \gtrsim 18 \pi\left(\frac{g_{\star} \mathcal{A}}{m_{\star}}\right)\left(\frac{g_{\star} \sigma}{m_{\star}}\right) .
$$

Suitable values for the inflaton mass $m_{\star}$ and couplings $\left\{g_{\star}, h_{\star}\right\}$ can be found so that this condition applies, but these choices are model-dependent. As an example, if we assume $h_{\star} \sim 10^{-1}$ and $m_{\star} \sim 10^{-5} M_{P}$, we find the universe reheats to a temperature

$$
T_{R} \simeq 1.3 \times 10^{-5} M_{P} \approx 3.12 \times 10^{13} \mathrm{GeV} .
$$

\subsection{Reheating temperatures}

The above analysis can be applied at once to the production mechanisms discussed in

$\S \S 3.13 .3$. Let us suppose that $\sigma \neq 0$, so that we are working with the symmetry-breaking potential. Assuming a typical coupling $g_{\star} \sim 1$, Eq. (4.2) implies that $\phi \rightarrow \chi \chi$ decay dominates resonant production when the amplitude becomes of order

$$
\mathcal{A} \lesssim 4 \times 10^{-4} \sigma\left(1-\frac{25 \epsilon}{64}\right)
$$

or smaller. The resulting reheating temperature receives relativistic corrections in comparison with the temperature achieved using canonical kinetic terms:

$$
T_{R} \simeq 0.13 \sigma\left(1-\frac{\epsilon}{8}\right) .
$$


On the other hand, decays into fermions will become predominant $(\phi \rightarrow \psi \bar{\psi})$ if the associated Yukawa coupling is sufficiently large

$$
h_{\star}^{2} \gtrsim 8 \pi\left(\frac{g_{\star} \sigma}{m_{\star}}\right)\left(\frac{g_{\star} \mathcal{A}}{m_{\star}}\right)\left(1+\frac{57 \epsilon}{64}\right) .
$$

This is compatible with the conditions $g \mathcal{A} / m \ll g \sigma / m \ll 1$. In this case, we find that, for a representative value of the coupling, say $h_{\star} \sim 0.1$

$$
T_{R} \simeq 3.12 \times 10^{13}\left(1-\frac{3 \epsilon}{8}\right) \mathrm{GeV}
$$

\section{Conclusions}

We have studied mechanisms by which the universe can be repopulated with matter species following an era of D-brane inflation. We work in the limit where the motion of the brane is at most perturbatively relativistic, which may ultimately be required by observation if $f_{\mathrm{NL}}$ measured on equilateral configurations of the bispectrum is nonnegative.

Our analysis is valid in a regime where the amplitude of oscillations is significantly smaller than whatever infrared scale caps the throat in which the D-brane moves. Indeed, because we take the D-brane to be at most perturbatively relativistic, inflation is of the slow-roll type. We assume inflation ends as the D-brane gently coasts to its resting position, dissipating its remaining energy by executing small amplitude oscillations. The amplitude and phase of these oscillations are mildly perturbated in comparison with the oscillations of canonically normalized scalar reheating the universe after a phase of four-dimensional inflation.

We encounter some novel effects which are not present in a theory with canonical kinetic terms. In canonical inflation there is no resonant production of inflaton particles during the reheating era. By contrast, in the perturbatively-relativistic limit of DBI inflation, nonlinearities arising from the Dirac-Born-Infeld action cause a weak resonance which reaches an $\mathrm{O}(1)$ effect only after $\sim 1 / \epsilon$ oscillations. Excessive production of inflaton particles is typically problematic, but since preheating typically concludes after $\lesssim 10$ oscillations this weak resonance is unlikely to be fatal for the DBI model. In the ultra-relativistic limit where $\gamma \gg 1$, the potential becomes unimportant and presumably resonance will be strongly suppressed.

Another novel effect is a 'DBI friction,' which appears in the equation of motion for the inflaton. This slightly damps the preheating effect. Beyond perturbation theory, it is possible this effect represents the decreasing significance of the potential at large $\gamma$.

For a given set of measured masses and couplings which characterize the properties of the inflaton and the matter field into which it decays, we find that final reheating tempera-

tures (assuming they are achieved by perturbative decays) are typically smaller than in the equivalent theory with canonical kinetic terms. This may be beneficial in concrete models, where lower reheating temperatures allow problems associated with overclosure of the universe by moduli or gravitinos to be ameliorated. The most stringent constraint on DBI models comes from observations of the microwave background bispectrum, which presently 
require $\gamma \lesssim 18$. If our results are representative, and the reheating temperature falls as $\gamma$ increases, a constraint on $\gamma$ may also emerge from demanding that the nucleosynthesis era is uninterrupted, which requires $T_{\mathrm{RH}} \gtrsim \mathrm{MeV}$.

\section{Acknowledgments}

NB is supported by the Wiener-Anspach Foundation. NB and DS acknowledge hospitality from DAMTP. ACD and DS acknowledge support from STFC. RHR is supported by FCT (Fundação para a Ciência e a Tecnologia - Portugal) through the grant SFRH/BD/35984/2007. We would like to thank Xingang Chen and David Tong for helpful discussions.

\section{A. Canonically normalized theory}

Consider a general higher-derivative Lagrangian of the form $P(\phi, X)$, given by Eq. (2.2). We allow small excitations $\delta \phi$ around the background field $\phi_{0}$. Assuming derivatives of $\phi_{0}$ are negligible there is a corresponding perturbation in the kinetic energy, $X=\delta \dot{\phi}^{2}-(\partial \delta \phi)^{2}$. To quadratic order in $\delta \phi$ but including all orders in other fields, the field propagates in a locally Minkowski region according to the action

$$
S=\int \mathrm{d}^{4} x\left\{P_{0}+\frac{\partial P}{\partial \phi} \delta \phi+\frac{\partial P}{\partial X}\left[\delta \dot{\phi}^{2}-(\partial \delta \phi)^{2}\right]+\frac{1}{2} \frac{\partial^{2} P}{\partial \phi^{2}} \delta \phi^{2}+\cdots\right\},
$$

where $P_{0}=P\left(X=0, \phi_{0}\right)$ and ' $\ldots$ ' denotes terms of higher order in $\delta \phi$ and its derivatives which have been omitted. Eq. (A.1) will give a reliable description of scattering and decay processes involving inflaton particles provided derivatives of the background field are negligible over the relevant time and distance scales. In this limit we can neglect the curvature of spacetime and work in a locally flat region, which should be chosen to be somewhat larger than the interaction region. Eq. (A.1) applies within this patch. S-matrix elements obtained from it can be expected to describe local scattering probabilities. On the other hand, if the background field $\phi$ varies significantly over the timescale of the scattering event then the definition of asymptotic in- and out-states becomes more complicated, and the concept of well-defined particles with an associated S-matrix may be invalidated. ${ }^{7}$

Canonical normalization. If the vacuum is locally stable on timescales comparable with decay processes, then $\langle\partial P / \partial \phi\rangle$ must be negligible when evaluated there. We must nevertheless retain perturbative excitations contained in this term, which include the fermion Yukawa couplings. After canonical normalization of $\delta \phi$, the interaction terms in the Lagrangian arise from

$$
\mathcal{L}_{\text {int }} \supseteq \frac{P_{, \phi}}{\sqrt{2 P_{, X}}} \delta \phi+\frac{1}{4} \frac{P_{, \phi \phi}}{P_{, X}} \delta \phi^{2} .
$$

where a comma denotes a partial derivative evaluated in the background. In a cut-off throat, the models we have been considering correspond to $P=-f^{-1}(1-f X)^{1 / 2}+f^{-1}-$

\footnotetext{
${ }^{7}$ For example, this may happen in models where $\phi_{0}$ evolves at a constant rate, such as the ghost inflation proposal of Arkani-Hamed et al. [47, 48]. However, this model is believed to suffer from certain pathologies.
} 
$V(\phi)$ with $f$ constant and $V(\phi)$ chosen to be Eq. (2.17) or (2.19). Consider the symmetrybreaking potential (2.19). We only wish to canonically normalize $\delta \phi$, which represents fluctuations around the mean field visible to local observers as particles. Therefore, one should shift to canonical normalization only after expanding in powers of fluctuations around the background field. Applying this procedure implies that we should make the transformations

$$
m^{2} \rightarrow m_{\star}^{2}=\frac{m^{2}}{2 P_{, X}} \quad \text { and } \quad g^{2} \rightarrow g_{\star}^{2}=\frac{g^{2}}{\sqrt{2 P_{, X}}} .
$$

Measurable masses and couplings. In this paper, we study the dynamics of particle production in different theories. If we are to make a meaningful comparison, these must be theories of the same particles. Therefore our results should be written in terms of the masses and couplings measured, for example, by a local observer who can record the outcome of scattering events.

In the case of non-canonical kinetic terms, it is possible that evolution of the background field causes the masses and couplings to change in time. In this case, it is the masses and couplings measured by a local observer at the time of reheating which are the relevant quantities.

After canonically normalizing the fluctuation $\delta \phi$, and working with tree-level scattering amplitudes, there are no further rescalings in the LSZ formula. Therefore, at tree-level, the coupling $g_{\star}$ of the canonically normalized field is the coupling which would be measured by experiment. Likewise, the mass $m_{\star}$ of the canonically normalized field determines the position of the pole in its propagator. It is therefore the quantities $m_{\star}$ and $g_{\star}$ which should be compared with the mass and coupling in a theory with canonical kinetic terms.

If a Yukawa coupling to fermions is present than we should similarly rescale its coupling constant,

$$
h \rightarrow h_{\star}=\frac{h}{\sqrt{2 P_{, X}}} .
$$

\section{B. Floquet exponents from Hill's equation}

In this appendix we derive the Floquet exponent associated with Hill's equation.

We start with the case when $\theta_{2}, \theta_{4} \neq 0$, and $\theta_{2 n}=0$, for $n>2$ and by noting that in the edges of the resonance bands $\mu_{k}=0$; it turns out that for the most important instability band and for $\theta_{2}, \theta_{4}<1$

$$
\theta_{0} \simeq 1 \pm \theta_{2}\left[1 \mp \frac{\left(\theta_{4}\right)^{2}}{6 \theta_{2}}\right],
$$

which, for $\theta_{4}^{2} / \theta_{2} \ll 1$, essentially reduces to

$$
\theta_{0} \simeq 1 \pm \theta_{2}
$$

From here, it also follows that, at lowest order, $\theta_{0}^{1 / 2} \simeq 1 \pm \theta_{2} / 2$. Furthermore, the instability parameter is given by 44

$$
\sin ^{2}\left(i \mu_{k} \frac{\pi}{2}\right)=\triangle(0) \sin ^{2}\left(\theta_{0}^{1 / 2} \frac{\pi}{2}\right)
$$


where $\triangle(0)$ is the discriminant evaluated for $\mu=0$ (following from Hill's method); $\triangle(0)$ is exclusively determined by the parameters $\theta_{0}, \theta_{2}$ and $\theta_{4}$ and is approximately given by

$$
\triangle(0) \simeq 1+\frac{\pi}{4 \theta_{0}^{1 / 2}} \cot \left(\theta_{0}^{1 / 2} \frac{\pi}{2}\right)\left[\frac{\theta_{2}^{2}}{1-\theta_{0}}+\frac{\theta_{4}^{2}}{4-\theta_{0}}\right] ;
$$

it can be shown that this, approximately, yields

$$
\triangle(0) \simeq 1 \mp\left(\frac{\pi}{2}\right)^{2}\left[\mp\left(\frac{\theta_{2}}{2}\right)^{2}+\frac{\theta_{2}}{3}\left(\frac{\theta_{4}}{2}\right)^{2}+\frac{\left(\theta_{2}\right)^{3}}{2}\right] .
$$

Using ( $(\overline{B .1})$ and $(\overline{B .3})$, we find that from $(\overline{B .2})$, we may arrive at an approximate expression for the instability parameter for this Hill's equation as

$$
\mu_{k} \simeq \sqrt{\left(\frac{\theta_{2}}{2}\right)^{2}-\left(\theta_{0}^{1 / 2}-1\right)^{2} \mp \frac{\theta_{2}}{3}\left(\frac{\theta_{4}}{2}\right)^{2} \mp \frac{\left(\theta_{2}\right)^{3}}{2}} .
$$

This expression for $\mu_{k}$ is valid for $\theta_{2}, \theta_{4} \ll 1$.

If we now proceed and consider the case with non-vanishing $\theta_{0}, \theta_{2}$ and $\theta_{6}$, we observe that the instability parameter may be computed using again using $(\overline{\mathrm{B} .2})$, where now

$$
\Delta(0) \simeq 1+\frac{\pi \cot \left(\frac{\pi}{2} \theta_{0}^{1 / 2}\right)}{4 \theta_{0}^{1 / 2}}\left(\frac{\theta_{2}^{2}}{1-\theta}+\frac{\theta_{6}^{2}}{9-\theta_{0}}\right) .
$$

By considering the first instability band, we take $\theta_{0} \simeq 1 \pm \theta_{2}$, so that

$\theta_{0}^{1 / 2} \simeq 1 \pm \frac{\theta_{2}}{2}, \frac{\theta_{2}^{2}}{1-\theta_{0}}=\mp \theta_{2} \quad, \quad \frac{\theta_{6}^{2}}{9-\theta_{0}} \simeq \mp \frac{\theta_{6}^{2}}{8}\left(1 \pm \frac{\theta_{2}}{8}\right) \quad$ and $\cot \left(\frac{\pi}{2} \theta_{0}^{1 / 2}\right) \simeq \mp \frac{\pi}{4} \theta_{2}$.

Plugging these into $(\overline{B .5})$, we find that, up to lowest order

$$
\Delta(0) \simeq 1 \mp\left(\frac{\pi}{4}\right)^{2}\left(\mp \theta_{2}^{2}+\frac{\theta_{2} \theta_{6}^{2}}{8}+\frac{\theta_{2}^{3}}{2}\right) .
$$

Moreover,

$$
\sin \left(\frac{\pi}{2} \theta_{0}^{1 / 2}\right) \simeq 1-\frac{1}{2}\left(\frac{\pi}{2}\right)^{2}\left(\theta_{0}^{1 / 2}-1\right)^{2}
$$

Finally, by substituting into (B.2), we obtain

$$
\mu_{k} \simeq \sqrt{\left(\frac{\theta_{2}}{2}\right)^{2} \mp \frac{\theta_{2} \theta_{6}^{2}}{32} \mp\left(\frac{\theta_{2}}{2}\right)^{3}-\left(\theta_{0}^{1 / 2}-1\right)^{2}} .
$$

\section{References}

[1] E. Komatsu et al., Seven-Year Wilkinson Microwave Anisotropy Probe (WMAP) Observations: Cosmological Interpretation, arXiv:1001.4538.

[2] X. Chen, Primordial Non-Gaussianities from Inflation Models, arXiv:1002.1416. 
[3] E. Silverstein and D. Tong, Scalar Speed Limits and Cosmology: Acceleration from Dcceleration, Phys. Rev. D70 (2004) 103505, arXiv:hep-th/0310221, doi:10.1103/PhysRevD.70.103505.

[4] M. Alishahiha, E. Silverstein, and D. Tong, DBI in the sky, Phys. Rev. D70 (2004) 123505, arXiv:hep-th/0404084, doi:10.1103/PhysRevD.70.123505.

[5] C. V. Johnson, D-branes, . Cambridge, USA: Univ. Pr. (2003) 548 p.

[6] L. McAllister and E. Silverstein, String Cosmology: A Review, Gen. Rel. Grav. 40 (2008) 565-605, arXiv:0710.2951, doi:10.1007/s10714-007-0556-6.

[7] X. Chen, Inflation from warped space, JHEP 08 (2005) 045, arXiv:hep-th/0501184].

[8] J. M. Cline, H. Firouzjahi, and P. Martineau, Reheating from tachyon condensation, JHEP 11 (2002) 041, arXiv:hep-th/0207156.

[9] J. H. Brodie and D. A. Easson, Brane inflation and reheating, JCAP 0312 (2003) 004, arXiv:hep-th/0301138, doi:10.1088/1475-7516/2003/12/004.

[10] G. Dvali, A. Gruzinov, and M. Zaldarriaga, Cosmological perturbations from inhomogeneous reheating, freezeout, and mass domination, Phys. Rev. D69 (2004) 083505, arXiv:astro-ph/0305548, doi:10.1103/PhysRevD.69.083505.

[11] A. R. Frey, A. Mazumdar, and R. C. Myers, Stringy effects during inflation and reheating, Phys. Rev. D73 (2006) 026003, arXiv:hep-th/0508139], ddoi:10.1103/PhysRevD.73.026003].

[12] D. Chialva, G. Shiu, and B. Underwood, Warped reheating in multi-throat brane inflation, JHEP 01 (2006) 014, arXiv:hep-th/0508229.

[13] L. A. Kofman, The origin of matter in the universe: Reheating after inflation, arXiv:astro-ph/9605155.

[14] D. I. Kaiser, Post inflation reheating in an expanding universe, Phys. Rev. D53 (1996) 1776-1783, arXiv:astro-ph/9507108, doi:10.1103/PhysRevD.53.1776.

[15] J. H. Traschen and R. H. Brandenberger, Particle production during out-of-equilibrium phase transitions, Phys. Rev. D42 (1990) 2491-2504, doi:10.1103/PhysRevD.42.2491.

[16] L. Kofman, A. D. Linde, and A. A. Starobinsky, Reheating after inflation, Phys. Rev. Lett. 73 (1994) 3195-3198, [arXiv:hep-th/9405187, doi:10.1103/PhysRevLett.73.3195.

[17] Y. Shtanov, J. H. Traschen, and R. H. Brandenberger, Universe reheating after inflation, Phys. Rev. D51 (1995) 5438-5455, arXiv:hep-ph/9407247, doi:10.1103/PhysRevD.51.5438.

[18] L. Kofman, A. D. Linde, and A. A. Starobinsky, Towards the theory of reheating after inflation, Phys. Rev. D56 (1997) 3258-3295, arXiv:hep-ph/9704452, doi:10.1103/PhysRevD.56.3258.

[19] R. Allahverdi, R. Brandenberger, F.-Y. Cyr-Racine, and A. Mazumdar, Reheating in Inflationary Cosmology: Theory and Applications, arXiv:1001.2600.

[20] L. Kofman, Preheating after inflation, Lect. Notes Phys. 738 (2008) 55-79, doi:10.1007/978-3-540-74353-8_.

[21] B. A. Bassett, S. Tsujikawa, and D. Wands, Inflation dynamics and reheating, Rev. Mod. Phys. 78 (2006) 537-589, arXiv:astro-ph/0507632, doi:10.1103/RevModPhys.78.537. 
[22] K. Kohri, D. H. Lyth, and C. A. Valenzuela-Toledo, On the generation of a non-gaussian curvature perturbation during preheating, JCAP 1002 (2010) 023, arXiv:0904.0793, doi:10.1088/1475-7516/2010/02/023.

[23] G. N. Felder et al., Dynamics of symmetry breaking and tachyonic preheating, Phys. Rev. Lett. 87 (2001) 011601, arXiv:hep-ph/0012142, doi:10.1103/PhysRevLett.87.011601.

[24] G. N. Felder, L. Kofman, and A. D. Linde, Tachyonic instability and dynamics of spontaneous symmetry breaking, Phys. Rev. D64 (2001) 123517, [arXiv:hep-th/0106179], doi:10.1103/PhysRevD.64.123517.

[25] J. F. Dufaux, G. N. Felder, L. Kofman, M. Peloso, and D. Podolsky, Preheating with Trilinear Interactions: Tachyonic Resonance, JCAP 0607 (2006) 006, arXiv:hep-ph/0602144.

[26] S. Kachru et al., Towards inflation in string theory, JCAP 0310 (2003) 013, arXiv:hep-th/0308055, doi:10.1088/1475-7516/2003/10/013.

[27] N. Barnaby, C. P. Burgess, and J. M. Cline, Warped reheating in brane-antibrane inflation, JCAP 0504 (2005) 007, arXiv:hep-th/0412040, doi:10.1088/1475-7516/2005/04/007.

[28] D. Baumann et al., On D3-brane potentials in compactifications with fluxes and wrapped D-branes, JHEP 11 (2006) 031, arXiv:hep-th/0607050.

[29] D. Baumann, A. Dymarsky, I. R. Klebanov, L. McAllister, and P. J. Steinhardt, A Delicate Universe, Phys. Rev. Lett. 99 (2007) 141601, arXiv:0705.3837, doi:10.1103/PhysRevLett.99.141601.

[30] D. Baumann, A. Dymarsky, I. R. Klebanov, and L. McAllister, Towards an Explicit Model of D-brane Inflation, JCAP 0801 (2008) 024, arXiv:0706.0360, doi:10.1088/1475-7516/2008/01/024.

[31] A. Krause and E. Pajer, Chasing Brane Inflation in String-Theory, JCAP 0807 (2008) 023, arXiv:0705.4682, doi:10.1088/1475-7516/2008/07/023.

[32] D. Baumann, A. Dymarsky, S. Kachru, I. R. Klebanov, and L. McAllister, Compactification Effects in D-brane Inflation, Phys. Rev. Lett. 104 (2010) 251602, arXiv:0912.4268].

[33] D. Baumann, A. Dymarsky, S. Kachru, I. R. Klebanov, and L. McAllister, D3-brane Potentials from Fluxes in AdS/CFT, JHEP 06 (2010) 072, [arXiv:1001.5028].

[34] D. A. Easson and R. Gregory, Circumventing the eta problem, Phys. Rev. D80 (2009) 083518, arXiv:0902.1798], doi:10.1103/PhysRevD.80.083518].

[35] L. Leblond and S. Shandera, Simple Bounds from the Perturbative Regime of Inflation, JCAP 0808 (2008) 007, arXiv:0802.2290, doi:10.1088/1475-7516/2008/08/007].

[36] S. Shandera, The structure of correlation functions in single field inflaiton, Phys. Rev. D79 (2009) 123518, arXiv:0812.0818], doi:10.1103/PhysRevD.79.123518].

[37] C. de Rham and A. J. Tolley, DBI and the Galileon reunited, JCAP 1005 (2010) 015, arXiv:1003.5917.

[38] D. N. Kabat and G. Lifschytz, Gauge theory origins of supergravity causal structure, JHEP 05 (1999) 005, arXiv:hep-th/9902073.

[39] L. Senatore, K. M. Smith, and M. Zaldarriaga, Non-Gaussianities in Single Field Inflation and their Optimal Limits from the WMAP 5-year Data, JCAP 1001 (2010) 028, arXiv:0905.3746, doi:10.1088/1475-7516/2010/01/028. 
[40] I. R. Klebanov and M. J. Strassler, Supergravity and a confining gauge theory: Duality cascades and chiSB-resolution of naked singularities, JHEP 08 (2000) 052, arXiv:hep-th/0007191.

[41] S. Kecskemeti, J. Maiden, G. Shiu, and B. Underwood, DBI inflation in the tip region of a warped throat, JHEP 09 (2006) 076, [arXiv:hep-th/0605189].

[42] C. Armendariz-Picon, M. Trodden, and E. J. West, Preheating in Derivatively-Coupled Inflation Models, JCAP 0804 (2008) 036, arXiv:0707.2177, doi:10.1088/1475-7516/2008/04/036.

[43] P. B. Greene and L. Kofman, On the theory of fermionic preheating, Phys. Rev. D62 (2000) 123516, arXiv:hep-ph/0003018, doi:10.1103/PhysRevD.62.123516.

[44] N. W. McLachlan, Theory and application of Mathieu functions. Oxford, UK: Clarendon Press (1947).

[45] F. M. Arscott, Periodic differential equations - an introduction to Lamé and allied functions. Oxford, UK: Pergamon Press (1964).

[46] A. J. Albrecht, P. J. Steinhardt, M. S. Turner, and F. Wilczek, Reheating an Inflationary Universe, Phys. Rev. Lett. 48 (1982) 1437, doi:10.1103/PhysRevLett.48.1437.

[47] N. Arkani-Hamed, H.-C. Cheng, M. A. Luty, and S. Mukohyama, Ghost condensation and a consistent infrared modification of gravity, JHEP 05 (2004) 074, arXiv:hep-th/0312099.

[48] N. Arkani-Hamed, P. Creminelli, S. Mukohyama, and M. Zaldarriaga, Ghost Inflation, JCAP 0404 (2004) 001, arXiv:hep-th/0312100, doi:10.1088/1475-7516/2004/04/001. 